\title{
IL-17 induces AKT-dependent IL-6/JAK2/STAT3 activation and tumor progression in hepatocellular carcinoma
}

Fang-Ming Gu${ }^{1 \dagger}$, Quan-Lin $\mathrm{Li}^{1,2+}$, Qiang Gao ${ }^{1 \dagger}$, Jia-Hao Jiang ${ }^{1}$, Kai Zhu ${ }^{3}$, Xiao-Yong Huang ${ }^{1}$, Jin-Feng Pan ${ }^{1}$, Jun Yan ${ }^{1}$, Jin-Hui Hu ${ }^{4}$, Zheng Wang ${ }^{1}$, Zhi Dai ${ }^{1,3}$, Jia Fan ${ }^{1,3}$ and Jian Zhou ${ }^{1,3^{*}}$

\begin{abstract}
Background: The Th17 subset and IL-17 have been found in increased frequencies within certain tumors. However, their relevance in cancer biology remains controversial. This study aimed to clarify the biological action of IL-17 on hepatocellular carcinoma (HCC).

Methods: Effects and underlying molecular mechanisms of IL-17 on human HCC were explored in vitro using exogenous IL-17 stimulation and in nude mice by implanting IL-17 overexpressed HCC cells. The clinical significance of IL-17 was investigated in tissue microarrays containing HCC tissues from 323 patients following hepatectomy using immunohistochemistry.
\end{abstract}

Results: Although exogenous IL-17 showed no direct effect on the growth rate of HCC cells in vitro, PCR and ELISA showed that IL-17 selectively augmented the secretion of diverse proinvasive factors and transwell showed a direct promotion of invasion of HCC cells by IL-17. Furthermore, transfection of IL-17 into HCC cells significantly promoted neoangiogenesis, neutrophil recruitment and tumor growth in vivo. Using siRNA mediated knockdown of AKT and STAT3, we suggested that the effects of IL-17 were operated through activation of the AKT signaling in HCC, which resulted in IL-6 production. Then, IL-6 in turn activated JAK2/STAT3 signaling and subsequently upregulated its downstream targets IL-8, MMP2, and VEGF. Supporting these findings, in human HCC tissues, immunostaining indicated that IL-17 expression was significantly and positively associated with STAT3 phosphorylation, neutrophil infiltration and increased tumor vascularity. The clinical significance of IL-17 was authenticated by revealing that the combination of intratumoral IL-17+ cells and phospho-STAT3 served as a better prognosticator for postoperative tumor recurrence than either marker alone.

Conclusions: IL-17 mediated tumor-promoting role involves a direct effect on HCC cells through IL-6/JAK2/STAT3 induction by activating the AKT pathway.

\section{Introduction}

Hepatocellular carcinoma (HCC) is the fifth most common cancer and the third most common cause of cancer-related death globally [1]. Despite advances in treatment modalities, long-term survival of HCC patients remains unsatisfactory because of the high rate of recurrence and metastasis [1]. HCC is usually secondary to inflammatory conditions due to chronic hepatitis and

\footnotetext{
* Correspondence: zhou.jian@zs-hospital.sh.cn

+ Contributed equally

'Liver Cancer Institute, Zhongshan Hospital, Fudan University, Shanghai, P.R. China
}

Full list of author information is available at the end of the article cirrhosis resulting from either hepatitis $\mathrm{B} / \mathrm{C}$ virus infection or from non viral-related causes such as alcohol or obesity. Compelling evidence has shown that inflammation orchestrates the microenvironment around $\mathrm{HCC}$, making a significant difference to cancer cell proliferation, migration, and survival [2].

T helper 17 (Th17) cells are an important inflammatory component whose main physiological role is to promote host defense against infectious agents, and are well appreciated for contributing to autoimmune diseases [3]. Recently, Th17 cells and its signature cytokine, interleukin-17 (IL-17), have been found increased frequencies 
within certain tumors [4-6]. However, the relationship between Th17 cells and tumor immunopathology has been controversial $[7,8]$. Both beneficial and detrimental direct and indirect effects of IL-17 occurred in context and tumor system dependent manners. Transfection of IL-17 into tumor cells augmented the progression of the disease in nude mice via the effects on vascular endothelium and increased neoangiogenesis $[9,10]$. In contrast, the same kind of experiments using syngeneic tumors in immunocompetent mice induced tumor suppression or even eradication by facilitating the recruitment of effector immune cells [11]. In clinical settings, a significant inverse correlation has been found between Th17 cell differentiation and prostate/ovarian cancer progression $[4,12]$, and low dose cyclophosphamide has newly been shown to modulate the tumor microenvironment by decreasing Treg suppressors while favoring Th17 and Th1 cells [13]. In HCC, IL-17+ T cells have been found in increased numbers within tumors and correlate with poor survival and increased postoperative recurrence, indicating that Th17 cells and IL-17 may promote tumor progression in HCC [14]. However, the direct effects and the underlying mechanisms of IL-17 in modulating human HCC cell growth remain elusive.

Previous studies have shown that IL-17 supported tumor progression via the effects on immune cells, vascular endothelial cells and stromal cells, focusing mostly on stimulating angiogenesis and inflammation. Given that many types of tumor cells bear IL-17 receptor alpha (IL17RA) $[15,16]$, the specific receptor for IL-17, IL-17 may have a direct impact on the biological behavior of tumor cells in the local microenvironment. As a confirmation, in murine B16 melanoma and MB49 bladder carcinoma, IL-17 mediated tumor-promoting role involves a direct effect on tumor cells through IL-6 induction and subsequent signal transducer and activator of transcription 3 (STAT3) activation [15]. IL-6 and other members of the IL-6 family of cytokines, including IL-11, in activating the JAK-STAT3 pathway leading to cancer-promoting inflammation has been widely documented [17]. It is well-known that cytokines' role in regulating tumor progression and metastasis are highly cell-type-dependent and context-dependent, highlighting that the effects of IL-17 on HCC cells mandate specific investigation. Thus, in this study, we attempted to elucidate the exact role and associated molecular mechanism of IL-17 in HCC proliferation and invasion in vitro and in nude mice. The clinical relevance and prognostic significance of IL-17 in human HCC were also investigated.

\section{Materials and methods \\ Cell lines}

Two human HCC cell lines, SMMC7721 (a human HCC cell line with low metastatic potential, established by the
Shanghai Institute of Cell Biology, Chinese Academy of Sciences, Shanghai, China) [18] and Huh7 (a well-differentiated and non-metastatic human HCC cell line, Japanese Cancer Research Resources Bank), were maintained in high-glucose Dulbecco's modified Eagle medium (DMEM) supplemented with $10 \%$ heat-inactivated fetal bovine serum (FBS), L-glutamine, 100 units $/ \mathrm{ml}$ penicillin, and $100 \mu \mathrm{g} / \mathrm{ml}$ streptomycin. All cell lines were cultured at $37^{\circ} \mathrm{C}$ in a humidified incubator in $5 \% \mathrm{CO}_{2}$.

\section{Quantitative reverse transcription-polymerase chain reaction (qRT-PCR)}

Total RNA was extracted with Trizol Reagent (Invitrogen, Carlsbad, CA) according to the manufacturer's protocol, and reversed transcribed with RevertAid ${ }^{\mathrm{TM}}$ first-strand cDNA synthesis kit (Fermentas, Burlington, ON). IL-17 and IL-17RA mRNA levels were determined by qPCR using SYBR Premix Ex Taq (TaKaRa, Dalian, China) and normalized with $\beta$-actin using the following primers: IL-17 forward, 5'-CGC TGA TGG GAA CGT GGA CTA C-3' and reverse, 5'-GGT GGA CAA TCG GGG TGA CA-3'. IL-17R forward, 5'-GTT CAT CAC GGG CAT CTC CAT C-3' and reverse, 5'-CAG GCA GGC CAT CGG TGT ATT-3'. $\beta$-actin forward, 5'-CAA CTG GGA CGA CAT GGA GAA AAT-3' and reverse, 5'-CCA GAG GCG TAC AGG GAT AGC AC-3'. The relative gene expression was calculated with the $2^{-\Delta \mathrm{Ct}}$ method.

\section{MTT, cell migration, and matrigel invasion assays}

The effect of IL-17 on HCC cell growth was determined with the 3-[4,5-dimethylthiazol-2-yl]-2,5-diphenyltetrazoliumbromide (MTT) assay. Cells were seeded into 96well flat-bottom plates $\left(1 \times 10^{3} /\right.$ well $)$ and cultured for 1 to 4 days in medium supplemented with recombinant human IL-17 $(0,0.1,0.5,1,5,10,50,100$, or $500 \mathrm{ng} / \mathrm{ml} ; 6$ wells/dose), and each experiment was repeated at least three times. After IL-17 treatment, cells were incubated with MTT $(20 \mu \mathrm{l} /$ well $)$ at $37^{\circ} \mathrm{C}$ for $4 \mathrm{~h}$, and then $200 \mu \mathrm{l}$ dimethylsulfoxide was added. The absorbance of individual wells was determined at $570 \mathrm{~nm}$.

The wound-healing assay was used to evaluate the ability of cell migration. Cells were grown to $80-90 \%$ confluence in 24-well plates, and a wound was made by dragging a plastic pipette tip across the cell surface. The remaining cells were washed three times to remove cell debris, and then incubated at $37^{\circ} \mathrm{C}$ with serum-free medium. After $48 \mathrm{~h}$, migrating cells at the wound front were photographed and compared. Three separate experiments were performed.

Cell invasion assays were performed using 24-well transwells $(8-\mu \mathrm{m}$ pore size; Minipore) precoated with Matrigel (BD Biosciences, San Jose, CA). In total, $1 \times 10^{5}$ cells, which were suspended in $100 \mu \mathrm{l}$ DMEM containing hIL-17 (50 ng/ml), 1\% FBS, IL-6 mAb (10 ng/ml), and/or IL-6 $(100 \mathrm{ng} / \mathrm{ml})$, were added to the upper chamber, and 
$600 \mu \mathrm{l}$ DMEM containing 10\% FBS was placed in the lower chamber. After $36 \mathrm{~h}$ of incubation, Matrigel and the cells remaining in the upper chamber were removed using cotton swabs. Cells on the lower surface of the membrane were fixed in $4 \%$ paraformaldehyde and stained with Giemsa. Cells in five microscopic fields (at $200 \times$ magnification) were counted and photographed. All experiments were performed in triplicate.

Recombinant human IL-17 and IL-6, and an IL-6 neutralizing $\mathrm{mAb}$ (R\&D Systems) were applied as appropriate.

\section{Immunofluorescence staining, ELISA, and western blot analysis}

For immunofluorescence staining, the monoclonal antibody (mAb) IL-17RA (R\&D Systems, Minneapolis, MN) was used.

The levels of IL-6, IL-1 $\beta$, TNF- $\alpha$, TGF- $\beta$, IL-8, G-CSF, GM-CSF, VEGF, and MMP2 in culture supernatants were measured by ELISA, following the manufacturer's instructions (R\&D Systems). Intra- and inter-assay coefficients of variation were $<5 \%$ and $<10 \%$, respectively.

Western blotting was performed as previously described [19]. The specific primary antibodies p-JAK2 (Y1007/ 1008), JAK2, p-STAT3 (Y705), STAT3, p-p65 NF- $\kappa$ B (S536), p65 NF- $\kappa \mathrm{B}, \mathrm{p}-\mathrm{AKT}$ (S473), AKT, p-JNK (T183/ Y185), JNK, p-ERK1/2 (T202/Y204), ERK1/2, p-p38 MAPK (T180/Y182) and p38 MAPK (Cell Signaling Technology, Beverly, MA), as well as MMP2 (Abcam, Cambridge, MA) were used. Glyceraldehyde-3-phosphate dehydrogenase (GAPDH; Millipore, Billerica, MA) was used as a loading control.

\section{Cell transfection and clone selection}

STAT3- and AKT-targeted siRNAs, as well as negative control mismatch sequences, were synthesized by Shanghai GeneChem Co. using the following sense and antisense strands: AKT sense, GUG CCA UGA UCU GUA UUU ATT and anti-sense, UAA AUA CAG AUC AUG GCA CTT; STAT3 sense, GGG ACC UGG UGU GAA UUA UTT and anti-sense, AUA AUU CAC ACC AGG UCC CTT. Transfection into SMMC7721 and Huh7 cells was performed using Lipofectamine ${ }^{\mathrm{TM}} 2000$ (Invitrogen) according to the manufacturer's instructions.

The pEGFP-N1-IL-17 plasmids were also constructed by Shanghai GeneChem Co. and pEGFP-N1 plasmids were used as controls. The lentiviral vector and plasmid were transfected into SMMC7721 cells as described elsewhere [20]. Stably transfected clones were validated by qRT-PCR and immunoblotting for the level of target gene expression (Additional file 1, Figure S1).

\section{Animal experiments}

$\mathrm{BALB} / \mathrm{ca}$ male nude mice (Shanghai Institute of Materia Medica, Chinese Academy of Science), 4 to 6 weeks old, were kept in laminar-flow cabinets under specific pathogen-free conditions, cared for, and handled according to the recommendations of the National Institutes of Health guidelines for care and use of laboratory animals. The experimental protocol was approved by Shanghai Medical Experimental Animal Care Committee. For the tumor challenge, $1 \times 10^{6}$ SMMC7721-IL-17 or SMMC7721mock tumor cells were injected subcutaneously into nude mice, and tumor growth was monitored each week [21]. There were five animals in each group. The mice were sacrificed on day 28 after tumor implantation. The levels of IL-6, IL-8, VEGF, and MMP2 in mice serum were measured by ELISA. Tumor tissues were prepared for Western blot analysis and immunostaining. Anti-mouse CD31 and Gr-1 mAbs (Abcam) were used for immunostaining.

\section{Patients and follow-up}

Tumor samples were obtained from 410 patients with pathologically confirmed $\mathrm{HCC}$ at Liver Cancer Institute of Zhongshan Hospital, Fudan University. Serial sections from 87 patients (cohort 1) treated between December 2010 and January 2011 were used for immunohistochemistry, among which 48, 21, and 18 patients were classified as TNM stage I, II, and III, respectively [22]. For tissue microarray (TMA) construction, archived tissues were obtained from 323 consecutive HCC patients treated between January 2003 and March 2004, with a median follow-up of 60 months (range, 2.0-74.0 months; cohort 2). All patients demonstrated no distant metastasis and had not received anticancer therapy before surgery. The tumor stage was determined according to the 2010 American Joint Committee on Cancer and International Union Against Cancer tumor-node-metastasis (TNM) classification system. Tumor differentiation was graded by the Edmondson grading system. The follow-up procedure was performed as described in our previous report [23]. Overall survival (OS) or time to recurrence was defined as the intervals between the dates of surgery and death or the first recurrence, respectively. Patients without death or recurrence were censored at the last follow up. Detailed clinicopathological characteristics are given in Table S1. Ethical approval was obtained from the research ethics committee of Zhongshan Hospital and written informed consent was obtained from each patient.

\section{Tissue microarrays and immunohistochemistry}

To examine the relationship between the densities of intratumoral IL-17+ cells and CD66b+ neutrophils or microvessel densities (MVDs), 87 cases (cohort 1) were chosen for immunohistochemistry using serial whole tumor sections.

TMAs were constructed as described elsewhere $[19,23]$ using tissues form cohort $2(n=323)$. Immunohistochemistry was conducted with the following 
primary mAbs: goat antihuman IL-17A (R\&D Systems), mouse antihuman CD66b (BD Pharmingen, San Diego, CA) and CD34 (Abcam), and rabbit antihuman p-STAT3 (Y705) (Cell Signaling Technology). Blank controls were treated identically with primary antibodies omitted.

The capture of the photographs and measurement of positive staining density were performed as described previously [23]. Briefly, for IL-17, p-STAT3, and CD66b staining in consecutive sections from $87 \mathrm{HCC}$ patients (cohort 1) and Gr-1 staining in xenografts, the sections were stained with indicated antibodies, and then were evaluated using light microscopy at $200 \times$ or $400 \times$ magnification by two investigators. Five representative fields of each case were captured. IL-17+ cell density or CD66b+ and Gr-1+ neutrophil density was evaluated according to the mean number of positive-staining cells in five count areas. For expression intensity of p-STAT3, the integrated absorbance and the area in a photograph were measured using Image-Pro Plus v6.0 software (Media Cybernetics, Inc.). A uniform setting of color segmentation was loaded for counting the integrated absorbance of all the pictures, and the mean p-STAT3 density was calculated as the product of the integrated absorbance/total area.

A Ki-67-specific monoclonal rat antibody (DAKO A/S) was used for detection and quantitation of tumor cell proliferation, while the In Situ Cell Death Detection kit-Peroxidase (Roche) was used for detection and quantitation of apoptosis. The Proliferation Index and Apoptosis Index were determined by calculating the number of Ki-67- or TUNEL-positive cells per total number of cells (hematoxylin-positive plus Ki-67- or TUNEL-positive cells) in 5 randomly selected fields at $\times 200$, using Image-Pro Plus v6.0 software as previously described [19].

For the MVD, sections were stained with CD31 or CD34 antibody and a diaminobenzidine reaction system for immunohistochemical assessment of tumor microvessels in five randomly selected fields at $200 \times$ magnification. The average number of microvessels was calculated. For the microvessel count, any brown-stained endothelial cell or endothelial cell cluster that was clearly separated from adjacent microvessels, tumor cells, and connective elements was counted as one microvessel, irrespective of the presence of a vessel lumen [4].

IL-17 and p-STAT3 staining in TMAs were evaluated at $200 \times$ magnification using light microscopy by two investigators blinded to the clinicopathologic data of the patients. For IL-17 immunostaining, the number of positive staining cells in each 1-mm diameter cylinder were calculated manually and expressed as the mean number of the duplicates (cells/1-mm core). For the expression intensity of p-STAT3, the integrated absorbance and the area in each $1-\mathrm{mm}$ diameter cylinder were measured using Image-Pro Plus v6.0 software. The mean p-STAT3 density was calculated as the product of the integrated absorbance/total area.

\section{Statistical analysis}

Statistical analysis was performed with SPSS 16.0 software (SPSS, Chicago, IL). Measurement values were expressed as means \pm standard deviations. Student's $t$ test, $\chi^{2}$ test, and Spearman's $\rho$ correlation were used as appropriate. The cumulative recurrence and survival rates were performed by the Kaplan-Meier method (log-rank test). Cox multivariate analysis with a stepwise method (forward, likelihood ratio) was used to determine the independent prognostic factors. Two-tailed $\mathrm{p}<0.05$ was judged to be significant.

The median value of IL-17+ cell counts was used as the cutoff to dichotomize IL-17 immunostaining. The optimal cutoff for dichotomizing p-STAT3 (Y705) expression data was determined using X-tile 3.6.1 software (Yale University of New Haven) [24].

\section{Results}

\section{IL-17 promotes HCC invasion and migration in vitro}

The expression of IL-17RA was easily detectable by qRT-PCR and immunofluorescence in Huh7 and SMMC7721 cells (Figure 1A). Because IL-17+ T cells were significantly elevated in HCC patients and correlated with poor survival [14], we thus assumed that IL17 could stimulate HCC cells through IL-17RA signaling pathways. Although IL-17 exerted little influence on cell proliferation (Additional file 2, Figure S2A), woundhealing assays revealed an evident increase in the wound closure rates of Huh7 and SMMC7721 cells after IL-17 $(50 \mathrm{ng} / \mathrm{ml})$ stimulation for $48 \mathrm{~h}$ (Figure 1B), and matrigel invasion assays showed that exogenous IL-17 (50 ng/ $\mathrm{ml}$ for $36 \mathrm{~h}$ ) significantly promoted the invasion of Huh7 and SMMC7721 cells (Figure 1C). These results indicated that IL-17 can promote invasion and migration of HCC in vitro.

\section{IL-17 up-regulates proinvasive factors in HCC in vitro}

IL-17 is known for eliciting secretion of diverse inflammatory mediators in diverse cell types, including stromal cells and tumor cells. We found that IL-17 selectively up-regulated the production of IL-6, IL-8, MMP2, and VEGF in Huh7 and SMMC7721 cells (Table 1). The most prominent secretion was IL-6, with 5.8- and 6.0fold increases, serially followed by IL-8 (2.5- and 5.6fold), MMP2 (1.5- and 1.6- fold) and VEGF (1.2- and 1.7- fold) in Huh7 and SMMC7721 cells respectively. By contrast, in Huh7 and SMMC7721 cells, the production of IL- $1 \beta$, TNF- $\alpha$, TGF- $\beta$, G-CSF and GM-CSF were not significantly affected by exogenous IL-17. 


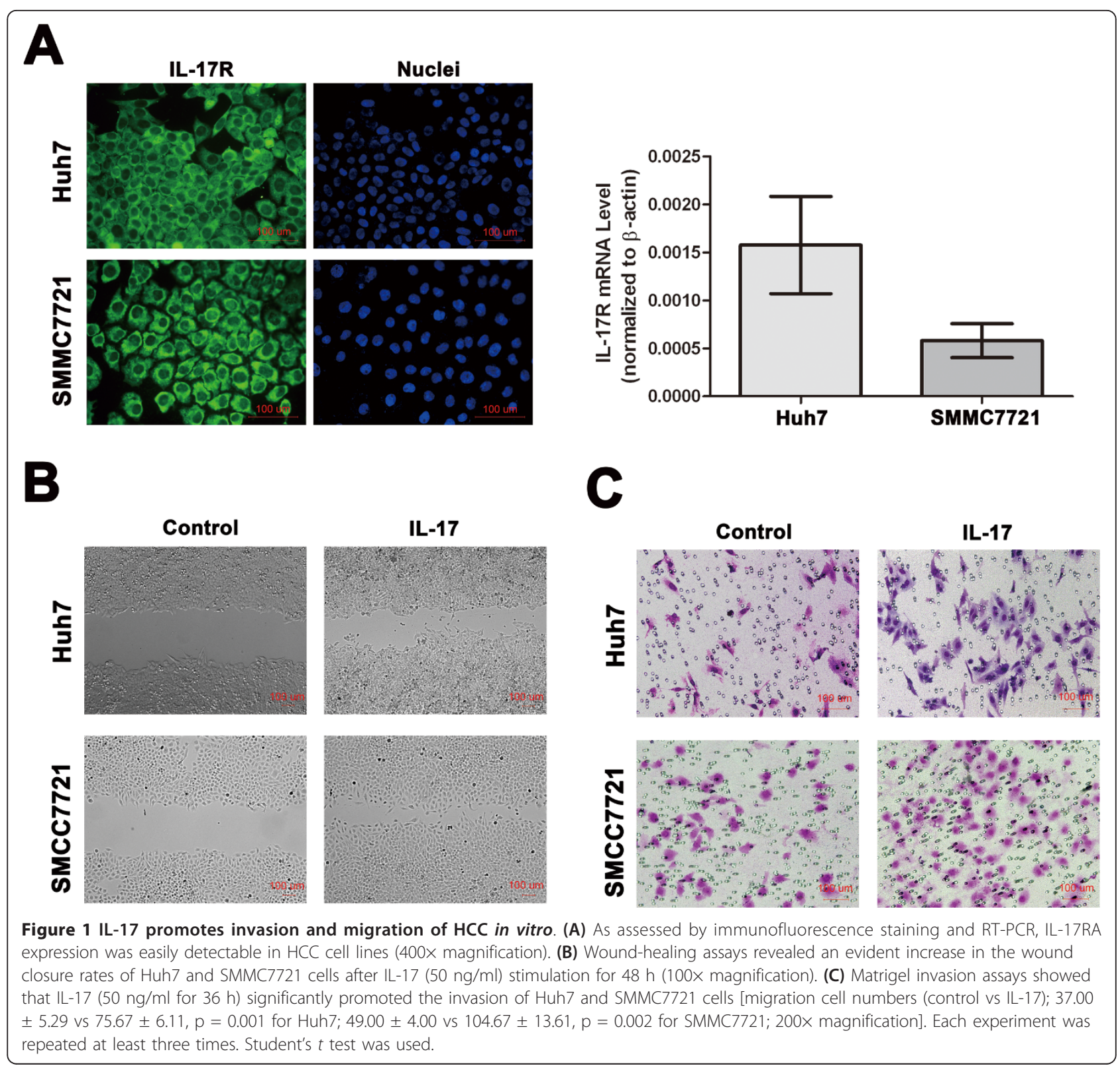

IL-17 activates STAT3 and AKT in HCC cells

Various signaling pathways are suggested to mediate IL17 action. Here, we scrutinized the potential signaling pathways of IL-17 action in HCC cells and found that IL-17 had no effect on p38 MAPK, ERK, JNK and p65 NF- $\kappa$ B activation in Huh7 and SMMC7721 cells (Additional file 2, Figure S2B). Intriguingly, phosphorylation of STAT3 and AKT were obviously increased as early as $3 \mathrm{~h}$ after IL-17 treatment and lasted for $24 \mathrm{~h}$ (Figure 2A, and Additional file 3, Figure S3A). Given that expression of IL-6, IL-8, MMP2, and VEGF increased in parallel with STAT3 and AKT activation in HCC cells treated with IL-17, STAT3, and AKT signaling may play important roles in inducing these proinvasive factors and hence tumor progression.

\section{IL-17 promotes HCC progression via AKT-dependent IL-6/ STAT3 activation}

To explore the potential role of STAT3 and AKT in IL17-mediated effects on HCC, STAT3 and AKT expression were reduced by small interfering RNA (siRNA).

First, in HCC cells exposed to STAT3-targeted siRNA (HCC-siRNA-STAT3), IL-17-induced AKT phosphorylation was not affected, while in $\mathrm{HCC}$ cells exposed to AKT-targeted siRNA (HCC-siRNA-AKT), IL-17-induced STAT3 phosphorylation was significantly reduced 
Table 1 IL-17 affects the production of inflammatory cytokines of HCC

\begin{tabular}{|c|c|c|c|c|c|c|}
\hline \multirow[b]{2}{*}{ IL-17 (50 ng/ml) } & \multicolumn{2}{|c|}{ Huh7 (pg/ml) } & \multirow[b]{2}{*}{$\mathrm{p}$} & \multicolumn{2}{|c|}{ SMMC7721 (pg/ml) } & \multirow[b]{2}{*}{$\mathrm{p}$} \\
\hline & - & + & & - & + & \\
\hline IL-6 & $7.0 \pm 1.5$ & $40.5 \pm 19.6$ & $<0.001$ & $63.3 \pm 33.8$ & $380.9 \pm 198.2$ & 0.001 \\
\hline IL-1 $\beta$ & $5.3 \pm 0.4$ & $5.53 \pm 0.5$ & 0.397 & $6.4 \pm 1.6$ & $5.6 \pm 1.0$ & 0.261 \\
\hline TNF- $\alpha$ & $22.5 \pm 2.3$ & $21.3 \pm 1.2$ & 0.206 & $22.8 \pm 3.3$ & $26.6 \pm 6.0$ & 0.137 \\
\hline TGF- $\beta$ & $215.7 \pm 51.9$ & $206.5 \pm 47.5$ & 0.716 & $140.7 \pm 83.2$ & $143.6 \pm 41.5$ & 0.931 \\
\hline IL-8 & $25.4 \pm 13.1$ & $141.3 \pm 133.9$ & 0.029 & $208.9 \pm 144.5$ & $521.7 \pm 208.3$ & 0.004 \\
\hline G-CSF & $10.9 \pm 1.2$ & $11.4 \pm 2.0$ & 0.575 & $20.3 \pm 14.9$ & $39.3 \pm 28.1$ & 0.112 \\
\hline GM-CSF & $6.5 \pm 0.5$ & $8.3 \pm 3.8$ & 0.212 & $8.40 \pm 2.23$ & $15.3 \pm 9.9$ & 0.078 \\
\hline VEGF & $2236.4 \pm 300.3$ & $3814.5 \pm 1188.6$ & 0.043 & $332.0 \pm 157.7$ & $557.8 \pm 162.2$ & 0.014 \\
\hline MMP2 & $327.6 \pm 12.2$ & $492.8 \pm 44.5$ & $<0.001$ & $351.4 \pm 14.3$ & $558.6 \pm 91.2$ & $<0.001$ \\
\hline
\end{tabular}

ELISA-determined cytokine levels were expressed as mean \pm SD. p-values were calculated by Student's $t$ test.

(Figure 2B and 2C, and Additional file 3, Figure S3B and S3C). Furthermore, IL-17-induced AKT phosphorylation was obviously increased as early as 5 minutes after IL17 treatment, while phosphorylation of JAK2 and STAT3 were not affected during the 60 minutes treatment (Figure 2D). These results suggested that AKT phosphorylation was an earlier event as compared with STAT3 phosphorylation in HCC stimulated with IL-17.
Additionally, in HCC-siRNA-STAT3 cells, IL-17induced expression of IL-8, MMP2, and VEGF were significantly inhibited, while IL-17-induced IL-6 upregulation was not affected (Figure 2B and 2E, and Additional file 3, Figure S3B and S3D). These results indicated that IL-8, MMP2, and VEGF were target genes instead of the upstream activators of STAT3. Furthermore, neutralizing IL-6 with a blocking mAb reduced JAK2 and

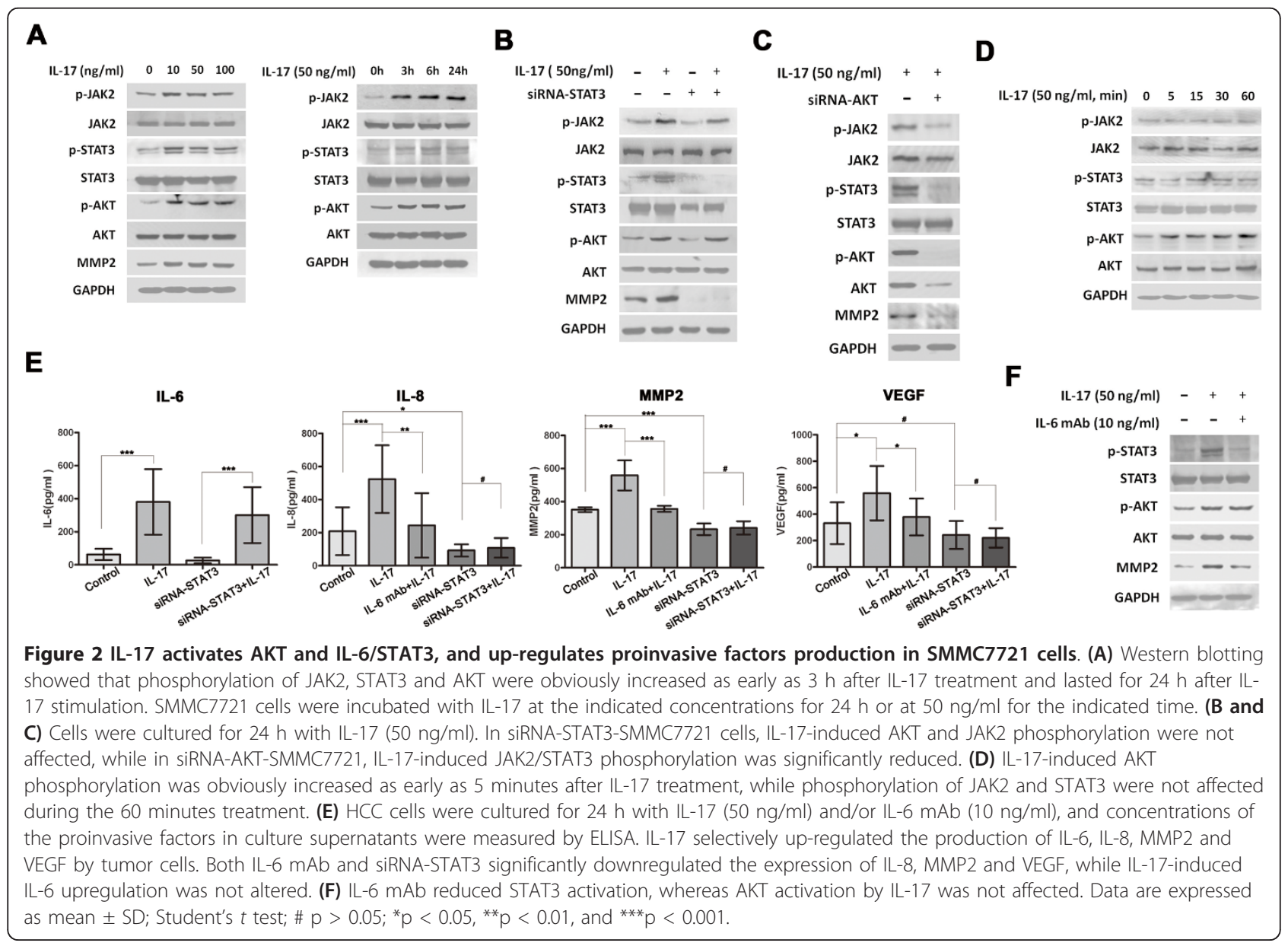


STAT3 activation and inhibited upregulation of IL-8, MMP2, and VEGF by IL-17, whereas AKT activation by IL-17 was not affected (Figure 2E and 2F, and Additional file 3, Figure S3D and S3E). Thus, IL-6 may be the main upstream activator, rather than the downstream target of JAK2/STAT3, in the setting of IL-17 stimulation.

By contrast, in HCC-siRNA-AKT cells, but not HCCsiRNA-STAT3 cells, IL-17-induced IL-6 upregulation was significantly blocked (Figure $2 \mathrm{E}$ and $3 \mathrm{~A}$, and Additional file 3, Figure S3D and Additional file 4, S4A). Thus, AKT siRNA both repressed IL- 6 and downregulated JAK2/STAT3 phosphorylation induced by IL-17 (Figure $2 \mathrm{C}, 3 \mathrm{~A}$ and $3 \mathrm{~B}$, and Additional file 3, Figure S3C, Additional file 4, S4A and S4B), indicating that IL-6/ JAK2/STAT3 may be the targets of AKT in the context of IL-17. Thus, IL-17 may upregulate IL-6 production via AKT activation. IL- 6 in turn activates JAK2/STAT3 and subsequently stimulates IL-8, MMP2, and VEGF production. Supporting this hypothesis, JAK2/STAT3 phosphorylation and the expression of IL-8, MMP2, and VEGF were significantly increased after addition of IL-17 plus exogenous IL-6 in siRNA-AKT-SMMC7721 and siRNA-AKT-Huh7 cells (Figure 3A and 3C, and Additional file 4, Figure S4A and S4C).

Meanwhile, STAT3-siRNA significantly reversed tumor invasion by IL-17 stimulation in HCC (Figure 3D, and Additional file 4, Figure S4D). An IL-6 neutralizing mAb reduced STAT3 activation and also completely reversed IL-17-stimulated tumor invasion in vitro (Figure 3D, and Additional file 4, Figure S4D), while exogenous IL-6 completely recovered IL-17-induced invasion of siRNA-AKTSMMC7721 and siRNA-AKT-Huh7 cells (Figure 3D, and Additional file 4, Figure S4D). AKT-dependent IL-6/ STAT3 activation was therefore suggested to be responsible for the tumor promoting effects of IL-17 on HCC.

\section{IL-17 promotes HCC progression in vivo}

When tumor cells were injected into nude mice, the growth rate of SMMC7721-IL-17 tumors was drastically

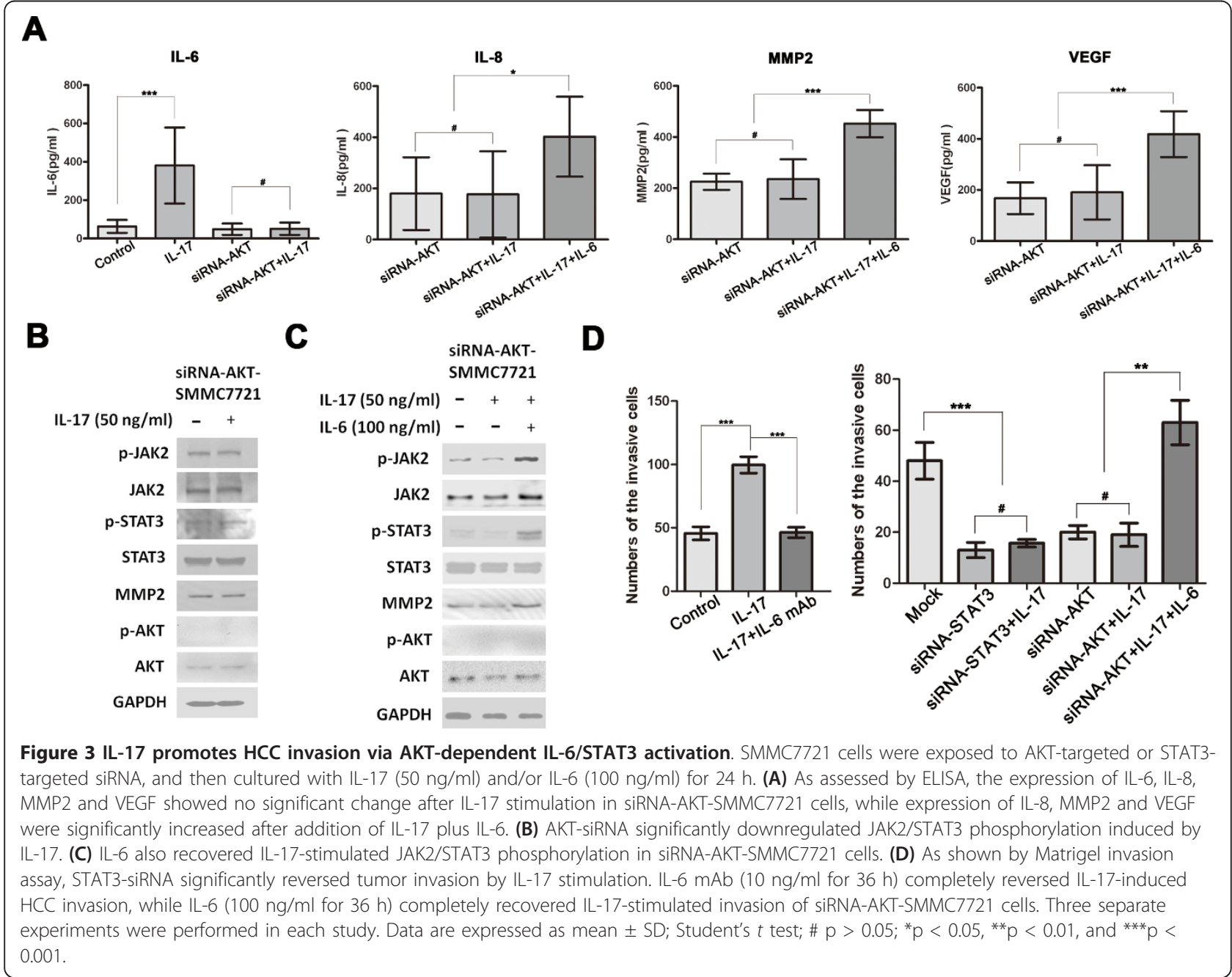


increased relative to SMMC7721-mock controls [mean tumor volume (mock vs IL-17), $0.589 \pm 0.259$ vs $4.175 \pm$ $3.050 \mathrm{~cm}^{3}, \mathrm{p}=0.031$; mean tumor weight (mock vs IL17), $0.392 \pm 0.124$ vs $2.140 \pm 0.963 \mathrm{~g}, \mathrm{p}=0.004$; respectively; Figure 4A]. Moreover, SMMC7721-IL-17-derived xenografts showed increased tumor cell proliferation while reduced apoptosis of HCC cells compared with the SMMC7721-mock group [proliferation index (mock vs IL-17), $71.260 \pm 9.584$ vs $83.820 \pm 6.549, \mathrm{p}=0.042$; apoptosis index (mock vs IL-17), $1.070 \pm 0.222$ vs 0.626 $\pm 0.320, \mathrm{p}=0.034$; respectively; Additional file 5 , Figure S5].

Increased phosphorylation of AKT was evident and the plasma concentration of IL-6 was significantly up-regulated in IL-17-transfected SMMC7721 mice compared with those in controls (Figure $4 \mathrm{~B}$ and $4 \mathrm{C}$ ). Overexpression of IL-17 also promoted JAK2/STAT3 activation and markedly up-regulated production of IL-8, MMP2, and VEGF (Figure 4B and 4C).

Apart from direct effects on tumor cells, MMP2 and VEGF are known for fostering angiogenesis and IL- 8 is a major chemotactic factor for neutrophil recruitment. Supporting this, SMMC7721-IL-17-derived xenografts presented with more neoangiogenesis (CD31+) and Gr-1 + neutrophil infiltration than the SMMC7721-mock group ( $<0.001$ for both; Figure 4D).

\section{Intratumoral IL-17+ cells positively correlate with p-STAT3} intensity, neutrophil and microvessel densities

Because in vivo SMMC7721-IL-17 tumors were characterized by increased angiogenesis and enriched neutrophil infiltration, we further explored whether a similar phenomenon exists in human HCC tissues. By staining consecutive sections in $87 \mathrm{HCC}$ patients (Figure 5A), a correlation analysis revealed that significant positive correlations were found between the density of intratumoral IL17 -producing cells and MVD $(\mathrm{r}=0.567 ; \mathrm{p}<0.001)$, as well as between the densities of intratumoral CD66b+ neutrophils and IL-17+ cells ( $r=0.630 ; p<0.001$; Figure 5B). Additionally, the p-STAT3 intensity significantly and positively correlated with levels of intratumoral IL-17+ cells, $\mathrm{CD66b}+$ neutrophils, and MVD $(\mathrm{r}=0.324$ and $\mathrm{p}=0.002 ; \mathrm{r}$ $=0.350$ and $\mathrm{p}<0.001 ; \mathrm{r}=0.544$ and $\mathrm{p}<0.001$; respectively; Additional file 6, Figure S6). Thus, IL-17+ cells may promote neoangiogenesis and neutrophil recruitment partly via STAT3 activation in human HCC.

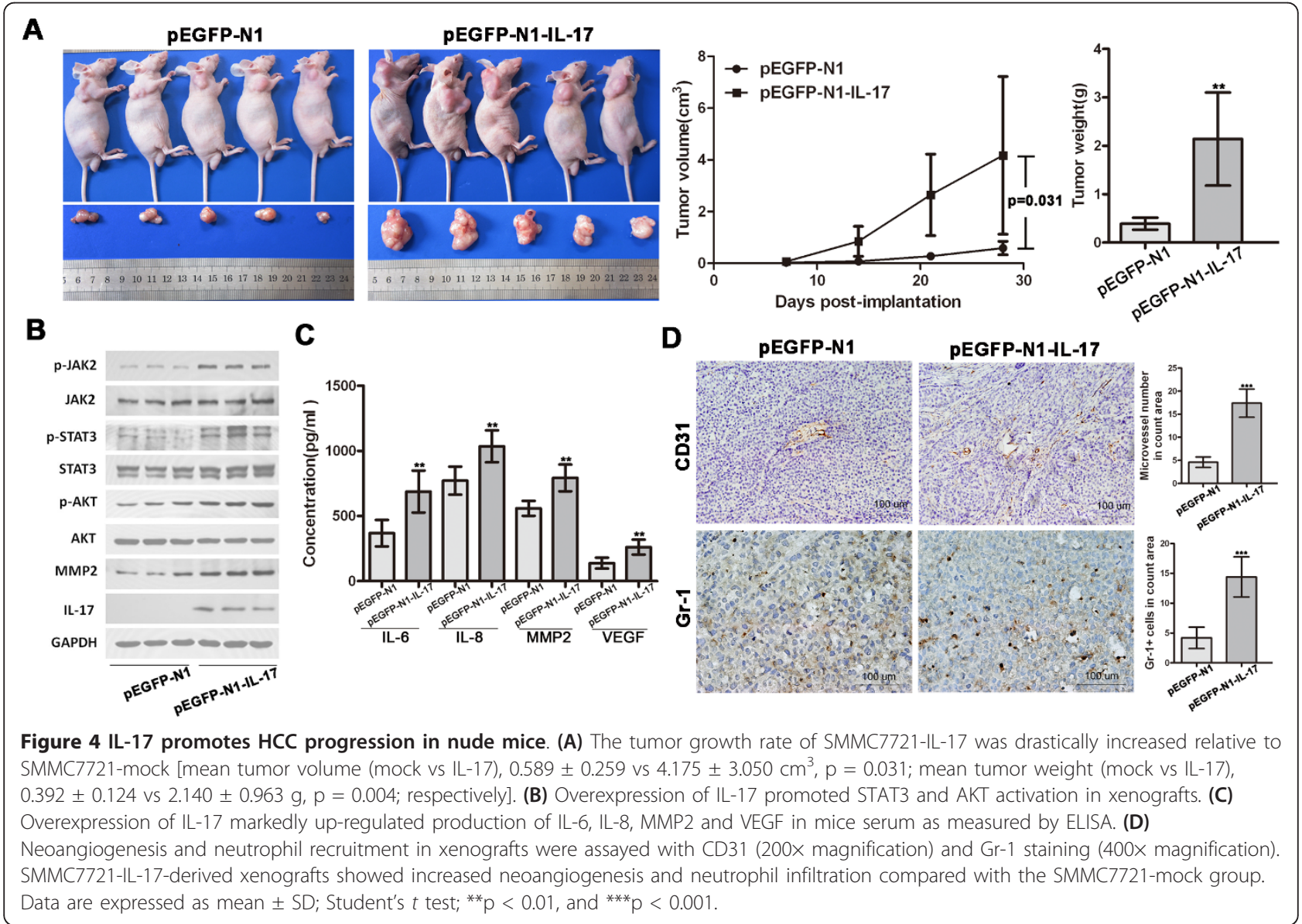




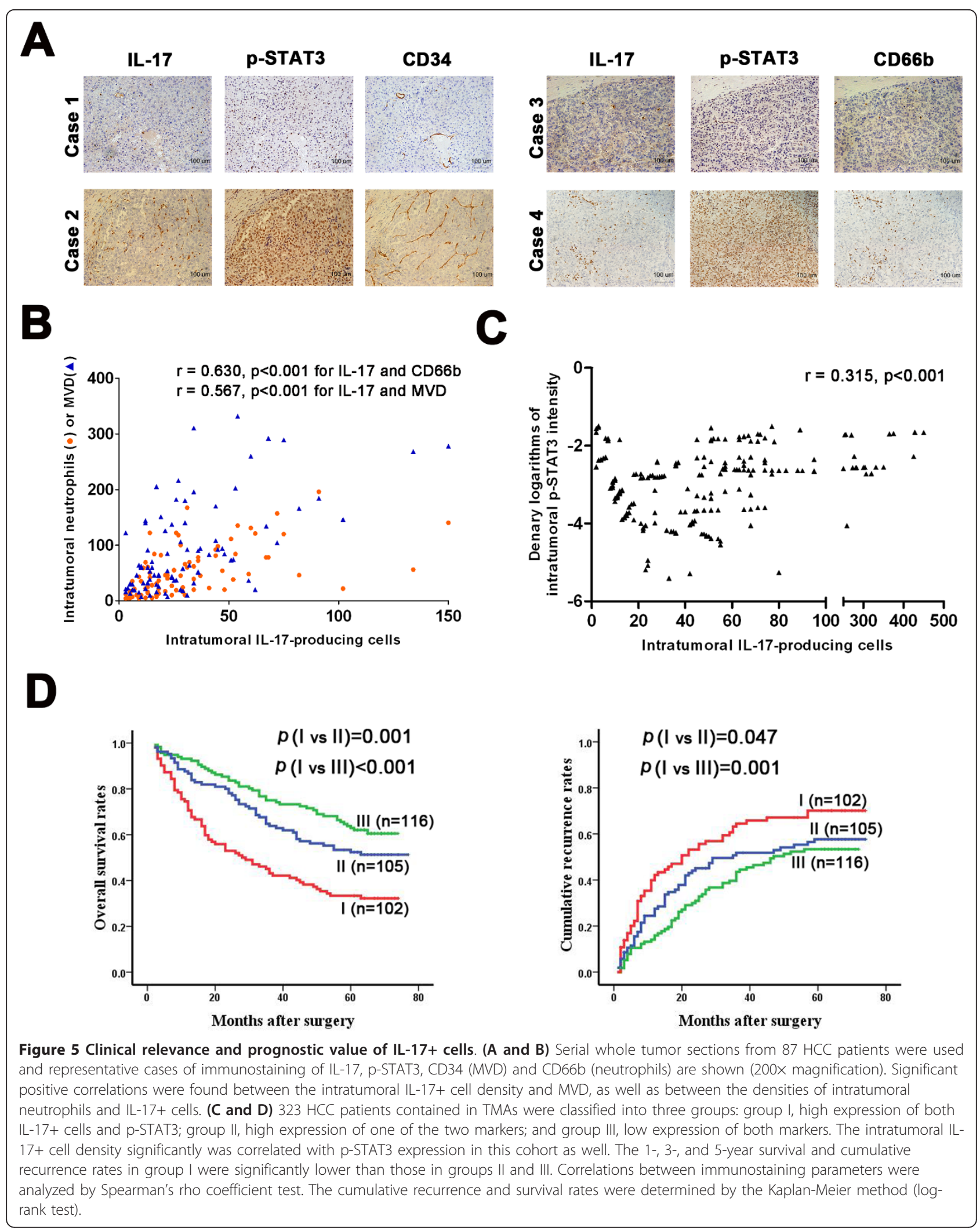


The combination of IL-17 and p-STAT3 is a better prognostic marker

We further investigated the prognostic value of IL-17 and its target, p-STAT3, both separately and in combination, in TMA containing $323 \mathrm{HCC}$ patients (Additional file 7, Figure S7). Similarly, the density of intratumoral IL-17+ cells significantly correlated with p-STAT3 expression in this cohort $(\mathrm{r}=0.315 ; \mathrm{p}<0.001$; Figure $5 \mathrm{C})$. Both IL$17^{\text {high }}$ and $\mathrm{p}-\mathrm{STAT} 3^{\text {high }}$ were significantly correlated with microvascular invasion $(\mathrm{p}=0.016$ and $\mathrm{p}=0.013$, respectively; Additional file 8, Table S1).

Among the 323 patients, the 5-year OS and cumulative recurrence rates were $50.7 \%$ and $59.7 \%$, respectively. Univariate analysis revealed that intratumoral IL-17+ cells and p-STAT3 expression were significantly associated with recurrence and survival (Table 2). Furthermore, patients were classified into three groups: group I, high expression of both markers; group II, high expression of either markers; and group III, low expression of both markers. Significant differences in recurrence and survival were detected among the three groups (Figure 5D).

Significant clinicopathologic features identified in univariate analysis were adopted as covariates in multivariate Cox models. Multivariate analyses showed that IL-17 + cell and p-STAT3 expression independently correlated with $O S$ and recurrence, irrespective of being used alone or in combination (Additional file 9, Table S2). However, concomitant high of IL-17 and p-STAT3 was superior to either marker alone in terms of hazard ratios and $\mathrm{p}$ values for both $\mathrm{OS}$ and recurrence (Additional file 9, Table S2). In multivariate analysis where IL-17+ cells, p-STAT3 expression and their combination were simultaneously adopted as covariates (Table 2), only their combination remained statistically significant. Specifically, compared with group III, hazard ratios of group I were 2.244 (95\% CI, 1.529-3.293; p < 0.001) for death and 1.718 (95\% CI, 1.193-2.473; $\mathrm{p}=0.004)$ for recurrence (Table 2 ), respectively.

\section{Discussion}

IL-17 has been shown to be elevated in several types of cancer, but how it might contribute to tumor growth is still unclear. Here, we found that IL-17 selectively augmented the secretion of various proinvasive factors and directly promoted in vitro invasion of HCC. Furthermore, transfection of IL-17 into HCC cells significantly promoted neoangiogenesis, neutrophil recruitment and tumor growth in vivo. These effects of IL-17 were suggested to be operated through activation of the AKT signaling, which resulted in IL-6 production. Then, IL-6 in turn activated JAK2/STAT3 signaling and subsequently up-regulated its downstream targets IL-8, MMP2, and VEGF. Supporting these findings, in human HCC tissues, IL-17 expression was significantly and positively associated with STAT3 phosphorylation, neutrophil infiltration, and increased tumor vascularity. Our findings thus

Table 2 Univariate and multivariate analyses of factors associated with survival and recurrence

\begin{tabular}{|c|c|c|c|c|c|c|}
\hline \multirow[b]{3}{*}{ Variables } & \multicolumn{3}{|c|}{ Overall survival } & \multicolumn{3}{|c|}{ Cumulative recurrence } \\
\hline & \multirow[b]{2}{*}{ Univariate: P } & \multicolumn{2}{|c|}{ Multivariate } & \multirow[b]{2}{*}{ Univariate: $\mathrm{P}$} & \multicolumn{2}{|c|}{ Multivariate } \\
\hline & & HR $(95 \% \mathrm{Cl})$ & $\mathbf{P}$ & & HR $(95 \% \mathrm{Cl})$ & $\mathbf{P}$ \\
\hline Age, years ( $\leq 51$ vs $>51$ ) & NS & NA & NA & NS & NA & NA \\
\hline Gender (female vs male) & 0.032 & NA & NS & 0.013 & $1.765(1.078-2.891)$ & 0.024 \\
\hline HBsAg (no vs yes) & NS & NA & NA & NS & NA & NA \\
\hline Liver cirrhosis (no vs yes) & NS & NA & NA & NS & NA & NA \\
\hline$\alpha$-Fetoprotein ( $\leq 20$ vs $>20 \mathrm{ng} / \mathrm{mL}$ ) & 0.016 & NA & NS & NS & NA & NA \\
\hline Tumor diameter $(\leq 5 \mathrm{vs}>5 \mathrm{~cm}$ ) & $<0.001$ & $2.192(1.583-3.035)$ & $<0.001$ & $<0.001$ & $1.613(1.183-2.200)$ & 0.003 \\
\hline Tumor number (single vs multiple) & 0.037 & NA & NS & 0.136 & NA & NS \\
\hline Tumor encapsulation (none vs complete) & 0.001 & $1.478(1.073-2.034)$ & 0.017 & 0.001 & $1.532(1.126-2.084)$ & 0.007 \\
\hline Tumor differentiation (I/II vs III/IV) & 0.009 & $1.581(1.118-2.237)$ & 0.010 & NS & NA & NA \\
\hline Vascular invasion (no vs yes) & $<0.001$ & $1.965(1.413-2.732)$ & $<0.001$ & $<0.001$ & $1.591(1.157-2.188)$ & 0.004 \\
\hline TNM stage (I vs II/III) & $<0.001$ & NA & NA & $<0.001$ & NA & NA \\
\hline IL-17 low vs IL-17 high & 0.001 & NA & NS & 0.018 & NA & NS \\
\hline p-STAT3 ${ }^{\text {low }}$ vs p-STAT3 high & $<0.001$ & NA & NS & 0.011 & NA & NS \\
\hline \multicolumn{7}{|l|}{ Combination of IL-17 and p-STAT3* } \\
\hline Overall & $<0.001$ & NA & $<0.001$ & $<0.001$ & NA & 0.009 \\
\hline I vs II & 0.001 & NA & NS & NS & NA & NS \\
\hline I vs III & $<0.001$ & $2.244(1.529-3.293)$ & $<0.001$ & 0.001 & $1.718(1.193-2.473)$ & 0.004 \\
\hline
\end{tabular}

Note: Univariate analysis was calculated using the Kaplan-Meier method (the log-rank test). Multivariate analysis was performed using the Cox multivariate proportional hazard regression model with a stepwise method (forward, likelihood ratio).

Abbreviations: HR, hazard ratio; Cl, confidence interval; HBsAg, hepatitis B surface antigen; NA, not assessed; NS, not significant; TNM, tumor-node-metastasis. *I, IL17 ${ }^{\text {high }}$ and p-STAT3 high; II, IL17 ${ }^{\text {high }}$ p-STAT3 ${ }^{\text {low }}$ or IL17 low p-STAT3 ${ }^{\text {high }}$; III, IL17 ${ }^{\text {low }}$ and p-STAT3 low 
support the notion that Th17 responses and IL-17 can promote HCC progression.

Previous studies have shown that IL-17 has diverse effects on inflammatory cells and stromal cells, focusing mostly on stimulating angiogenesis and inflammation [9-11]. Recently, in primary hepatocytes, IL-17 was shown to up-regulate a group of inflammatory cytokine genes, most of which are NF- $\kappa \mathrm{B}$ target genes [25]. Given that the expression of IL-17RA was considerable in HCC cells, we thus hypothesize that IL-17-mediated tumor-promoting role involved a direct effect on HCC cells. Supporting this, we found that exogenous IL-17 directly promoted HCC cell invasion in vitro and enhances tumor progression in vivo. Although consistent with several recent publications regarding the role of IL-17 in promoting tumor growth, these findings contradict other reports suggesting that IL17 can provide an antitumor effect against certain tumors $[4,11]$

The means by which IL-17 achieves its effects, either for the benefit or the detriment of the host, are largely due to the induction of new gene expression. However, different cell types appear to respond differently to IL-17 in terms of target gene expression. More recently, IL-17 has been also reported to mediate the release of proinflammatory factors and chemokines from tumor cells, including renal, lung and cervical cancers $[10,26,27]$. Here, we showed that IL-17 selectively up-regulated the production of proinvasive factors in HCC cells, including IL-6, IL-8, MMP2, and VEGF. As master regulators of cell dissemination, proliferation and angiogenesis, the production of these factors from HCC cells inevitably resulted in the progression of HCC. By contrast, although GM-CSF, G-CSF, TNF- $\alpha$, TGF- $\beta$ and IL- $1 \beta$ have been reported to be affected by IL17 in other cell types [28], production of these cytokines in HCC cells was not significantly altered under IL-17.

IL-17 mediates signaling through distinct pathways in numerous inflammatory cells and tumor cells, such as MAPK, NF- $\kappa$ B, and STAT3 pathways [16]. We found that IL-17 significantly induced STAT3 and AKT phosphorylation, but had no obvious effect on p38 MAPK, ERK, JNK and p65 NF- $\kappa$ B activation in HCC cells. These indicated that STAT3 and AKT activation play an important role in the induction of proinvasive factors and hence tumor progression. Previously, IL-17 has been reported to stimulate production of IL- 6 and STAT3 activation in inflammatory cells and fibroblasts in an autoimmune disease [29], as well as in cancer cells $[15,30]$. Furthermore, IL-6 mediated activation of STAT3 in tumor cells results in increases in anti-apoptotic, pro-proliferation, and pro-angiogenic genes [31]. In this study, using siRNA-mediated AKT and STAT3 knockdown, our data further extended these findings by indicating that IL-17 stimulated IL-6 induction was attributed to the activation of the AKT pathway; IL-6 in turn activated JAK2/STAT3 and up-regulated the transcription of the proinvasive genes. Our results were consistent with the hypothesis that, under certain conditions, AKT signaling may be the new proximal signaling mediator used by the IL-17R family to mediate downstream events $[16,32,33]$. To the best of our knowledge, this study is the first to demonstrate that IL-17induced IL-6/STAT3 activation was dependent on the activation of the AKT signaling pathway in tumor cells.

Because previous studies suggested the opposite role of IL-17 in tumor systems in nude mice versus immunocompetent mice [9-11], we further verified whether our finding in the xenograft nude mouse HCC model could be reproducible in human HCC. We found that intratumoral IL-17-producing cells were positively and significantly correlated with intratumoral neutrophils and MVD, not only in transplanted HCC model but also in human HCC tissues. The results supported that aside from direct promotion effects on tumor invasion and migration, IL-17 could also promote tumor angiogenesis and neutrophil recruitment through induction of HCC cells to releasing angiogenic and chemotactic factors such as IL-8, MMPs and VEGF. Alternatively, IL-17 may directly affect vascular endothelial cells and recruite circulating neutrophils by serving as neutrophil chemotactic and angiogenic factors, which were correlated with poor prognosis in HCC $[34,35]$. Furthermore, the levels of IL-17+ cells positively correlated with the expression of p-STAT3, both of which were associated with the presence of microvascular invasion, and poor survival in HCC patients. The combination of intratumoral IL-17+ cells and p-STAT3 expression served as a better predictor than either used alone in HCC patients.

\section{Conclusions}

In conclusion, our results suggested that the IL-17 mediated tumor-promoting role involved a direct effect on tumor cells through IL-6 induction by activating the AKT pathway; IL-6 in turn activated JAK2/STAT3 and up-regulated proinvasive factors IL-8, MMP2, and VEGF both in vitro and in vivo. Therapies that target IL-17 and STAT3 may be developed as potential therapeutic approaches to inhibit HCC.

\section{Additional material}

Additional file 1: Figure S1 SMMC7721 cells are stably transfected with lentiviral-mediated pEGFP-N1-IL-17 plasmids. The pEGFP-N1-IL17 plasmids were constructed and pEGFP-N1 plasmids were used as controls. The lentiviral vector and plasmid were transfected into SMMC7721 cells. SMMC7721 cells were successfully transfected with pEGFP-N1-IL-17 plasmids validated by fluorescent imaging (A), QRT-PCR, and immunoblotting (B) for the level of IL-17 expression.

Additional file 2: Figure S2 IL-17 shows no effect on tumor proliferation, as well as p38 MAPK, ERK, JNK, and p65 NF- $\kappa$ B 
activation in vitro. (A) IL-17 had no effect on tumor proliferation as assessed by the MTT assay. Cells were cultured for 1 to 4 days in medium supplemented with IL-17 $(0,0.1,0.5,1,5,10,50,100$ or $500 \mathrm{ng} /$ $\mathrm{ml})$. (B) Huh7 and SMMC7721 cells were incubated with IL-17 (50 ng/ml) for the indicated time. As assessed by Western blot analysis, IL-17 showed no effect on p38 MAPK, ERK, JNK, and p65 NF- $\kappa$ B activation.

Additional file 3: Figure S3 IL-17 activates AKT and IL-6/STAT3, and up-regulates proinvasive factors production in Huh7 cells. (A) Western blotting showed that phosphorylation of JAK2, STAT3 and AKT were obviously increased as early as $3 \mathrm{~h}$ after $\mathrm{IL}-17$ treatment and lasted for $24 \mathrm{~h}$ after IL-17 stimulation. Huh7 cells were incubated with IL-17 at the indicated concentrations for $24 \mathrm{~h}$ or at $50 \mathrm{ng} / \mathrm{ml}$ for the indicated time. (B and C) Cells were cultured for $24 \mathrm{~h}$ with $\mathrm{IL}-17(50 \mathrm{ng} / \mathrm{ml})$. In siRNA-STAT3-Huh7 cells, IL-17-induced AKT and JAK2 phosphorylation were not affected, while in siRNA-AKT- Huh7, IL-17-induced JAK2/STAT3 phosphorylation was significantly reduced. (D) HCC cells were cultured for $24 \mathrm{~h}$ with IL-17 $(50 \mathrm{ng} / \mathrm{ml})$ and/or IL-6 mAb $(10 \mathrm{ng} / \mathrm{ml})$, and concentrations of the proinvasive factors in culture supernatants were measured by ELISA. IL-17 selectively up-regulated the production of IL-6, IL-8, MMP2 and VEGF by tumor cells. Both IL-6 mAb and siRNA-STAT3 significantly downregulated the expression of IL-8, MMP2 and VEGF, while IL-17-induced IL-6 upregulation was not altered. (E) IL-6 mAb reduced STAT3 activation, whereas AKT activation by IL-17 was not affected.

Additional file 4: Figure S4 IL-17 promotes HCC invasion via AKTdependent IL-6/STAT3 activation. Huh7 cells were exposed to AKTtargeted or STAT3-targeted siRNA, and then cultured with IL-17 (50 ng/ $\mathrm{ml}$ ) and/or IL-6 (100 $\mathrm{ng} / \mathrm{ml})$ for $24 \mathrm{~h}$. (A) As assessed by ELISA, the expression of IL-6, IL-8, MMP2 and VEGF showed no significant change after IL-17 stimulation in siRNA-AKT-Huh7 cells, while expression of IL-8, MMP2 and VEGF were significantly increased after addition of IL-17 plus IL-6. (B) AKT-siRNA significantly downregulated JAK2/STAT3 phosphorylation induced by IL-17. (C) IL-6 also recovered IL-17stimulated JAK2/STAT3 phosphorylation in siRNA-AKT-Huh7 cells. (D) As shown by Matrigel invasion assay, STAT3-siRNA significantly reversed tumor invasion by IL-17 stimulation. IL-6 mAb $(10 \mathrm{ng} / \mathrm{ml}$ for $36 \mathrm{~h})$ completely reversed IL-17-induced HCC invasion, while IL-6 $(100 \mathrm{ng} / \mathrm{ml}$ for $36 \mathrm{~h}$ ) completely recovered IL-17-stimulated invasion of siRNA-AKTHuh7 cells. Three separate experiments were performed in each study.

Additional file 5: Figure S5 IL-17 promotes tumor cell proliferation and inhibites apoptosis of HCC cells in nude mice. Proliferation index and apoptosis index in xenografts were assayed with Ki-67 and TUNEL staining (200x magnification). SMMC7721-IL-17-derived xenografts showed increased tumor cell proliferation while reduced apoptosis of HCC cells compared with the SMMC7721-mock group.

Additional file 6: Figure S6 The intratumoral p-STAT3 intensity is positively correlated with IL-17+ cell, neutrophil and microvessel densities. Serial whole tumor sections from 87 HCC patients were used for immunostaining. The intratumoral p-STAT3 staining intensity was significantly and positively correlated with the levels of intratumoral IL-17 + cells, neutrophils, and MVD. Correlations between immunostaining parameters were analyzed by Spearman's rho coefficient test.

Additional file 7: Figure S7 Representative cases of immunohistochemical staining of IL-17 and p-STAT3 in a tissue microarray. Consecutive sections of case 1 and case 2 showed high $1 \mathrm{~L}-$ $17+$ cells and p-STAT3 staining, while consecutive sections of case 3 and 4 showed low IL-17+ cells and p-STAT3 staining.

Additional file 8: Table S1 Correlation between IL-17 or p-STAT3 and clinicopathological characteristics. Both $\mathrm{LL}-17^{\text {high }}$ and p-STAT3 ${ }^{\text {high }}$ were significantly correlated with microvascular invasion.

Additional file 9: Table S2 Multivariate analyses of prognosis factors associated with survival. IL-17+ cell and p-STAT3 expression independently correlated with OS and recurrence, irrespective of being used alone or in combination. However, concomitant high of IL-17 and p-STAT3 was superior to either marker alone in terms of hazard ratios and $p$ values for both $O S$ and recurrence.

\section{Acknowledgements}

National Natural Science Foundation of China (30972949 and 30901432), Shanghai Rising-Star Program (10QA1401300), Research Fund for the Doctoral Program of Higher Education of China (No. 20090071120026), and National Key Sci-Tech Special Project of China (2008ZX10002-025).

\section{Author details}

${ }^{1}$ Liver Cancer Institute, Zhongshan Hospital, Fudan University, Shanghai, P.R. China. ${ }^{2}$ Endoscopy Center and Endoscopy Research Institute, Zhongshan Hospital, Fudan University, Shanghai, P.R.China. Institute of Biomedical Sciences, Fudan University, Shanghai, P.R.China. ${ }^{4}$ Department of Laboratory Medicine, Gongli Hospital, Pudong new area, Shanghai, P.R.China.

\section{Authors' contributions}

JZ conceived and supervised the study. FG, QL, QG performed in vivo and in vitro experiments, imaging studies and data analysis. FG, QL, QG, JJ, KZ collected clinical data. JH, ZW, ZD, JF assisted with in vivo and in vitro studies. FG, QL, QG drafted the manuscript. All authors read, edited and approved the manuscript.

\section{Competing interests}

The authors declare that they have no competing interests.

Received: 16 June 2011 Accepted: 15 December 2011

Published: 15 December 2011

\section{References}

1. El-Serag HB, Rudolph KL: Hepatocellular carcinoma: epidemiology and molecular carcinogenesis. Gastroenterology 2007, 132:2557-2576.

2. Ferrone $C$, Dranoff G: Dual roles for immunity in gastrointestinal cancers. J Clin Oncol 2010, 28:4045-4051.

3. Miossec P, Korn T, Kuchroo VK: Interleukin-17 and type 17 helper T cells. N Engl J Med 2009, 361:888-898.

4. Kryczek I, Banerjee M, Cheng P, Vatan L, Szeliga W, Wei S, Huang E, Finlayson E, Simeone D, Welling TH, Chang A, Coukos G, Liu R, Zou W: Phenotype, distribution, generation, and functional and clinical relevance of Th17 cells in the human tumor environments. Blood 2009, 114:1141-1149

5. Steiner GE, Newman ME, Paikl D, Stix U, Memaran-Dagda N, Lee C, Marberger MJ: Expression and function of pro-inflammatory interleukin IL-17 and IL-17 receptor in normal, benign hyperplastic, and malignant prostate. Prostate 2003, 56:171-182.

6. Zhang B, Rong G, Wei H, Zhang M, Bi J, Ma L, Xue X, Wei G, Liu X, Fang G The prevalence of Th17 cells in patients with gastric cancer. Biochem Biophys Res Commun 2008, 374:533-537.

7. Bronte V: Th17 and cancer: friends or foes? Blood 2008, 112:214

8. Murugaiyan G, Saha B: Protumor vs antitumor functions of IL-17. J Immunol 2009, 183:4169-4175.

9. Numasaki M, Fukushi J, Ono M, Narula SK, Zavodny PJ, Kudo T, Robbins PD, Tahara H, Lotze MT: Interleukin-17 promotes angiogenesis and tumor growth. Blood 2003, 101:2620-2627.

10. Tartour E, Fossiez F, Joyeux I, Galinha A, Gey A, Claret E, Sastre-Garau X, Couturier J, Mosseri V, Vives V, Banchereau J, Fridman WH, Wijdenes J, Lebecque S, Sautes-Fridman C: Interleukin 17, a T-cell-derived cytokine, promotes tumorigenicity of human cervical tumors in nude mice. Cancer Res 1999, 59:3698-3704.

11. Benchetrit F, Ciree A, Vives V, Warnier G, Gey A, Sautes-Fridman C, Fossiez F, Haicheur N, Fridman WH, Tartour E: Interleukin-17 inhibits tumor cell growth by means of a T-cell-dependent mechanism. Blood 2002, 99:2114-2121.

12. Sfanos KS, Bruno TC, Maris CH, Xu L, Thoburn CJ, DeMarzo AM, Meeker AK, Isaacs WB, Drake CG: Phenotypic analysis of prostate-infiltrating lymphocytes reveals TH17 and Treg skewing. Clin Cancer Res 2008, 14:3254-3261.

13. Viaud S, Flament C, Zoubir M, Pautier P, LeCesne A, Ribrag V, Soria JC, Marty V, Vielh P, Robert C, Chaput N, Zitvogel L: Cyclophosphamide induces differentiation of Th17 cells in cancer patients. Cancer Res 2011, 71:661-665. 
14. Zhang JP, Yan J, Xu J, Pang XH, Chen MS, Li L, Wu C, Li SP, Zheng L: Increased intratumoral IL-17-producing cells correlate with poor survival in hepatocellular carcinoma patients. J Hepatol 2009, 50:980-989.

15. Wang L, Yi T, Kortylewski M, Pardoll DM, Zeng D, Yu H: IL-17 can promote tumor growth through an IL-6-Stat3 signaling pathway. J Exp Med 2009, 206:1457-1464.

16. Gaffen SL: Structure and signalling in the IL-17 receptor family. Nat Rev Immunol 2009, 9:556-567.

17. Yu H, Pardoll D, Jove R: STATs in cancer inflammation and immunity: a leading role for STAT3. Nat Rev Cancer 2009, 9:798-809.

18. Ye QH, Qin LX, Forgues M, He P, Kim JW, Peng AC, Simon R, Li Y, Robles Al, Chen Y, Ma ZC, Wu ZQ, Ye SL, Liu YK, Tang ZY, Wang XW: Predicting hepatitis B virus-positive metastatic hepatocellular carcinomas using gene expression profiling and supervised machine learning. Nat Med 2003, 9:416-423.

19. Gao Q, Wang XY, Qiu SJ, Yamato I, Sho M, Nakajima Y, Zhou J, Li BZ, Shi YH, Xiao YS, Xu Y, Fan J: Overexpression of PD-L1 significantly associates with tumor aggressiveness and postoperative recurrence in human hepatocellular carcinoma. Clin Cancer Res 2009, 15:971-979.

20. Shi GM, Ke AW, Zhou J, Wang XY, Xu Y, Ding ZB, Devbhandari RP Huang XY, Qiu SJ, Shi YH, Dai Z, Yang XR, Yang GH, Fan J: CD151 modulates expression of matrix metalloproteinase 9 and promotes neoangiogenesis and progression of hepatocellular carcinoma. Hepatology 2010, 52:183-196.

21. Fu J, Chen Y, Cao J, Luo T, Qian YW, Yang W, Ren YB, Su B, Cao GW, Yang $Y$, Yan YQ, Shen F, Wu MC, Feng GS, Wang HY: p28GANK overexpression accelerates hepatocellular carcinoma invasiveness and metastasis via phosphoinositol 3-kinase/AKT/hypoxia-inducible factor1alpha pathways. Hepatology 2011, 53:181-192.

22. Berasain C, Castillo J, Perugorria MJ, Latasa MU, Prieto J, Avila MA: Inflammation and Liver Cancer. Annals of the New York Academy of Sciences 2009, 1155:206-221.

23. Gao Q, Qiu SJ, Fan J, Zhou J, Wang XY, Xiao YS, Xu Y, Li YW, Tang ZY: Intratumoral balance of regulatory and cytotoxic $T$ cells is associated with prognosis of hepatocellular carcinoma after resection. J Clin Oncol 2007, 25:2586-2593.

24. Camp RL, Dolled-Filhart M, Rimm DL: X-tile: a new bio-informatics tool for biomarker assessment and outcome-based cut-point optimization. Clin Cancer Res 2004, 10:7252-7259.

25. Sparna T, Retey J, Schmich K, Albrecht U, Naumann K, Gretz N, Fischer HP, Bode JG, Merfort I: Genome-wide comparison between IL-17 and combined TNF-alpha/IL-17 induced genes in primary murine hepatocytes. BMC Genomics 2010, 11:226.

26. Inozume T, Hanada K, Wang QJ, Yang JC: IL-17 secreted by tumor reactive T cells induces IL-8 release by human renal cancer cells. J Immunother 2009, 32:109-117.

27. Numasaki M, Watanabe M, Suzuki T, Takahashi $H$, Nakamura A, McAllister $F$, Hishinuma T, Goto J, Lotze MT, Kolls JK, Sasaki H: IL-17 enhances the net angiogenic activity and in vivo growth of human non-small cell lung cancer in SCID mice through promoting CXCR-2-dependent angiogenesis. J Immunol 2005, 175:6177-6189.

28. Onishi RM, Gaffen SL: Interleukin-17 and its target genes: mechanisms of interleukin-17 function in disease. Immunology 2011, 129:311-321.

29. Ogura H, Murakami M, Okuyama Y, Tsuruoka M, Kitabayashi C, Kanamoto M, Nishihara M, Iwakura $Y$, Hirano T: Interleukin-17 promotes autoimmunity by triggering a positive-feedback loop via interleukin-6 induction. Immunity 2008, 29:628-636.

30. Wang L, Yi T, Zhang W, Pardoll DM, Yu H: IL-17 enhances tumor development in carcinogen-induced skin cancer. Cancer Res 2010, 70:10112-10120.

31. Yu H, Kortylewski M, Pardoll D: Crosstalk between cancer and immune cells: role of STAT3 in the tumour microenvironment. Nat Rev Immunol 2007, 7:41-51.

32. Huang F, Kao CY, Wachi S, Thai P, Ryu J, Wu R: Requirement for both JAKmediated PI3K signaling and ACT1/TRAF6/TAK1-dependent NF-kappaB activation by IL-17A in enhancing cytokine expression in human airway epithelial cells. J Immunol 2007, 179:6504-6513.

33. Hwang SY, Kim JY, Kim KW, Park MK, Moon Y, Kim WU, Kim HY: IL-17 induces production of IL-6 and IL- 8 in rheumatoid arthritis synovial fibroblasts via NF-kappaB- and PI3-kinase/Akt-dependent pathways. Arthritis Res Ther 2004, 6:R120-128.
34. Roussel L, Houle F, Chan C, Yao Y, Berube J, Olivenstein R, Martin JG, Huot J, Hamid Q, Ferri L, Rousseau S: IL-17 promotes p38 MAPKdependent endothelial activation enhancing neutrophil recruitment to sites of inflammation. J Immunol 2010, 184:4531-4537.

35. Li YW, Qiu SJ, Fan J, Zhou J, Gao Q, Xiao YS, Xu YF: Intratumoral neutrophils: a poor prognostic factor for hepatocellular carcinoma following resection. J Hepatol 2010, 54:497-505.

doi:10.1186/1476-4598-10-150

Cite this article as: Gu et al.: IL-17 induces AKT-dependent IL-6/JAK2/ STAT3 activation and tumor progression in hepatocellular carcinoma. Molecular Cancer 2011 10:150.

\section{Submit your next manuscript to BioMed Central and take full advantage of:}

- Convenient online submission

- Thorough peer review

- No space constraints or color figure charges

- Immediate publication on acceptance

- Inclusion in PubMed, CAS, Scopus and Google Scholar

- Research which is freely available for redistribution

Submit your manuscript at www.biomedcentral.com/submit
Biomed Central 